\title{
Modelo de estrategia didáctica para fortalecer el aprendizaje de matemática en estudiantes de segundo bachillerato, unidad educativa vicente rocafuerte, Ecuador-2020
}

\author{
Magister Gómez Samaniego Gardenia Marisol \\ https://orcid./ 0000-0003-2127-3801 \\ p7001262519@ucvvirtual.edu.pe
}

Magister Cayambe Guachilema Miltón Doroteo

https://orcid./ 0000-0003-4772-894X

p7001262556@ucvvirtual.edu.pe

Magister Bermudez Pacheco Manuel Valentin

https://orcid./0000-0003-3582-0079

p7001266895@ucvvirtual.edu.pe

Magister Nuñez Michuy Carlos Manuel

https://orcid./0000-0003-2298-7697

cnunesz@ueb.edu.pe

Universidad César Vallejo

Piura - Perú

\section{RESUMEN}

La presente investigación tuvo como objetivo proponer un Modelo de estrategia didáctica para fortalecer el aprendizaje de matemática en estudiantes de segundo bachillerato de la Unidad Educativa Vicente Rocafuerte de Ecuador. Se utilizó la metodología de enfoque cuantitativo de tipo descriptivo propositivo y con un diseño no experimental. Para el diagnóstico sobre el uso de las TAC en las clases de matemáticas se realizó un cuestionario aplicado a 40 estudiantes objeto de la investigación a través de un formulario de Google, y para identificar las estrategias para fortalecer la enseñanza de las matemáticas se utilizó la revisión de documentos de los últimos 5 años de repositorios digitales. Teniendo como resultado la propuesta de un modelo de estrategias didácticas fundamentado en las teorías del constructivismo basado en los aportes de Ausubel y Vigotsky, el Conectivismo, el método de Polya, considerando además el entorno digital actual. Concluyendo que es necesario el uso de las herramientas tecnológicas, donde existe una avalancha de información que se encuentra al alcance de todos, aspecto, lo que 
debería ser aprovechado por los docentes, adaptando esta información de manera estratégica y didáctica para facilitar el aprendizaje matemático donde el docente tendría el rol fundamental de orientador de la información para lograr su asimilación.

Palabras clave: conectivismo; heurística; gamificación; geogebra; tecnologías 


\title{
Didactic strategy model to strengthen mathematics learning in second high school students, vicente rocafuerte educacional unit, Ecuador-2020
}

\begin{abstract}
The objective of this research was to propose a didactic strategy model to strengthen the learning of mathematics in second-year students of the Vicente Rocafuerte Educational Unit of Ecuador. The methodology of quantitative approach of a descriptive proposition type and with a non-experimental design was used. To diagnose the use of CT in mathematics classes, a questionnaire was applied to 40 students object of the research through a Google form, and to identify the strategies to strengthen the teaching of mathematics, the review was used of documents from the last 5 years of digital repositories. Resulting in the proposal of a model of didactic strategies based on the theories of constructivism based on the contributions of Ausubel and Vygotsky, Connectivism, Polya's method, also considering the current digital environment. Concluding that the use of technological tools is necessary, where there is an avalanche of information that is available to everyone, aspect, which should be used by teachers, adapting this information in a strategic and didactic way to facilitate mathematical learning where the teacher would have the fundamental role of guiding the information to achieve its assimilation.
\end{abstract}

Keywords: connectivism; heuristics; gamification; geogebra; technologies

Artículo recibido: 10 Setiembre. 2021 Aceptado para publicación: 15 Octubre. 2021 Correspondencia: mmm_marisol@yahoo.es Conflictos de Interés: Ninguna que declarar 


\section{INTRODUCCIÓN}

De acuerdo por lo establecido por la UNESCO (2021a) indica que los maestros de la asignatura de Matemáticas movilizan las expectativas, gustos e intereses hacia el curso, para ello debe promover situaciones problemáticas con exigencias coherentes a la edad del estudiante, que al enfrentarlas o resolverlas ellos expongan " $\mathrm{Si}$, eso yo lo puedo resolver". El ir involucrándolo constantemente en buscar la resolución, fomenta que resuelvan problemas con mayor nivel de exigencia. Lo expuesto anteriormente fundamenta que el éxito radica en enseñar aprovechando el error, aprendiendo del error. De acuerdo a lo planteado líneas arriba se hace necesario conocer los resultados del dominio de las matemáticas a nivel de América Latina en donde la UNESCO (2021b) indica que el nivel de rendimiento de las matemáticas es bajo por ejemplo en alumnos de sexto de primaria, el $62 \%$ de alumnos están ubicados en el nivel bajo, concentrándose el mayor porcentaje en tareas sencillas, como: estimación de peso y longitud, identificación de posición relativa en planos, mapas, identificación de patrón o regla de creación de secuencia numérica simple, resolución de problemas sencillos y lectura de datos evidentes en tabla y gráfico. En otro aspecto, se evidencia que este grupo de alumnos presentan dificultad en las habilidades de: relación de diversas direcciones en el espacio, encontrar el término faltante, encontrar el ángulo, identificar, comprobar la medida de longitud, masa, volumen, etc.

La Unión Europea considera a las matemáticas como una destreza clave para la formación personal, equidad en la sociedad y aptitudes para encajar en el ámbito laboral, y debido al bajo desempeño educativo debieron formularse un objetivo para suplir esta deficiencia y se proyectaron al 2020 que el bajo rendimiento académico en matemáticas debe ser menor que el 15\%. Para lograr esto Europa reforma el diseño curricular en matemáticas, para lograr esto plantea metodología educativa y de evaluación con innovación, formación docente mejorada desde su etapa inicial, y apoyo a los educadores para que mejores sus destrezas matemáticas y sobre todo la metodología para resolver problemas, con esto apuesta a la reducción reveladora de un mal rendimiento escolar. (Romo et al., 2020, p.98)

Asimismo, en los resultados realizado por el Programa para la Evaluación Internacional de Estudiantes (PISA), quien evalúa a los alumnos según las competencias logradas en el área de matemática a los estudiantes de 15 años, que se encuentren en el último grado de 
la educación básica, son considerada como evaluaciones internacionales, ejecutadas cada tres años, participando países miembros de la OCDE. En donde se indica que en Latinoamérica la proporción de estudiantes que lograron el nivel dos y los primeros lugares en la Región los puntajes de estos varían entre 50,7\% (Chile) y 9,4\% (República Dominicana). (PISA, 2020)

Para el caso de Ecuador esta realidad no ajena toda vez que de acuerdo al Comercio (2019) indica que el área en que tienen más inconvenientes es Matemáticas, concluyendo que existen en esta área grandes brechas que se deben ir mejorando, básicamente esto implica que se implementen estrategias para poder comprender el proceso de enseñanza aprendizaje en los alumnos. Por otro lado, entre las causas por el bajo rendimiento en matemáticas por parte de los alumnos de la institución objeto de investigación, es tal vez un currículo centrado en concretar temas y avanzar contenidos, esta forma acelerada de aprendizaje genera conflicto en la atención y concentración del estudiantes, asimismo encontramos la parte del docente en la cual los métodos escasos para gestionar en los alumnos sesiones de aprendizaje amenas, motivantes para inducir un mejor desempeño matemático, evidencia obstáculo en el aprendizaje y aumente la cantidad de alumnos que tienes desagrado por el aprendizaje matemático. (Alarcón, Flores, 2021, p. 36).

A este panorama del acápite anterior debemos agregarle que muchos docentes del área de matemáticas son profesionales en otras ramas entre ellos se encuentran los ingenieros, contadores, economistas quienes poseen dominio y conocimiento numérico pero carecen del conocimiento pedagógico y didáctico, los cual influye en el bajo rendimiento académico que obtienen los educandos, al ser profesionales de otras áreas se basan en resultados, directos a dar solución a ejercicios propuestos, en muchos casos los problemas formulados no tiene desempeño auténtico, muchos educadores no relacionados con pedagogía optaron por una maestría en educación pero esta formación no es suficiente para adquirir estrategias, metodologías y sobre todo sólidos conocimientos pedagógicos, en relación a esta fenómeno muchos estudiantes manifiestan que no comprenden la explicación que el docentes realiza sobre un tema y que mientras más explica lo que genera es mayor confusión.

Por lo descrito en los párrafos anteriores en donde se vislumbra la problemática de las variables se hace necesario la siguiente formulación del problema: ¿De qué manera el 
modelo de estrategia didáctica fortalece el aprendizaje de matemática en estudiantes de segundo bachillerato, Unidad Educativa Vicente Rocafuerte, Ecuador-2020?

Esta investigación se justifica teóricamente debido a que nos permite conocer la problemática sobre el aprendizaje de la matemática, en este sentido es de vital importancia comprender las teorías que la sustentan, además de comprender las teorías que permitan mejorar la práctica docente. Por otro lado, la justificación práctica radica en que los resultados de la investigación serán manifestados a las autoridades de la institución para tomar medidas correctivas y buscar solucionar los problemas del aprendizaje de las matemáticas, por otro lado, también se considera un aporte la estrategia didáctica como parte fundamental en el proceso de mejorar la práctica docente y por ende la enseñanza del conocimiento matemático. Finalmente, la justificación metodológica se centra en el aporte del modelo de estrategia didáctica y además del instrumento que permite medir el aprendizaje de las matemáticas el mismo que ha sido validado por expertos, mostrando un nivel de confiabilidad alto y por lo tanto está apto para ser aplicado en otras instituciones.

A propósito de lo descrito es pertinente plantearse el objetivo general que es: Proponer un modelo de estrategia didáctica que fortalece el aprendizaje de matemática en estudiantes de segundo bachillerato, Unidad Educativa Vicente Rocafuerte, Ecuador2020. Asimismo, los objetivos específicos tenemos: a) Diagnosticar el uso de las TAC en las clases de matemáticas en estudiantes de segundo bachillerato, Unidad Educativa Vicente Rocafuerte, Ecuador-2020. b) identificar estrategias para fortalecer el aprendizaje de matemáticas c) Diseñar, modelo de estrategia didáctica que fortalece el aprendizaje de matemática en estudiantes de segundo bachillerato, Unidad Educativa Vicente Rocafuerte, Ecuador-2020

\section{2- METODOLOGÍA.}

\section{Tipo y diseño de investigación.}

\section{Tipo de investigación}

Esta investigación utilizó el enfoque cuantitativo, ya que realiza una recolección de datos, porque todos los datos que se obtienen se convertirán en información, los cuales podrán ser cuantificados y apreciados en la estadística. Valderrama (2013). Es propositiva porque diseña una propuesta en donde aplica instrumentos y técnicas estadísticas para el proceso e interpretación de la información. 


\section{Diseño de investigación.}

El diseño de investigación es no experimental, no se sometió a ningún sistema de prueba. (Hernández \& Mendoza, 2018) nos indican que la investigación no experimental observa fenómenos para analizarlos, no generan situaciones sino observan situaciones que ya existen. El diseño se representa de la siguiente manera:

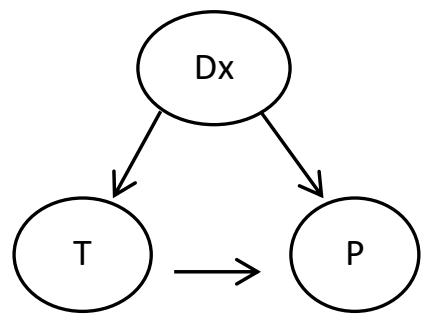

\section{En dónde:}

Dx: Descripción de realidad.

T: Conocimiento de bases teóricas

P. Propuesta de estrategia didáctica

\section{Variables y operacionalización.}

En relación a la variable aprendizaje de matemática tenemos que en el proceso de operacionalización se desintegro en dimensiones e indicadores, para posteriormente construir el instrumento de recojo de datos. Frente a este procedimiento se ha considerado que las dimensiones que han surgido son ámbito académico y recursos tecnológicos.

Por consiguiente, en función a la variable estrategia didáctica se realizó una revisión documental para identificar las estrategias utilizadas en la enseñanza de las matemáticas y buscar teorías que las sustenten en para la propuesta del modelo para fortalecer el aprendizaje de las matemáticas.

\section{Población, muestra y muestreo}

\section{Población}

Siguiendo el aporte de (Gamarra et al., 2008) considera a la población como "la totalidad de los elementos, que están definidos, delimitados y viables de los cuales se tomará una referencia para seleccionar la muestra estimando una serie de criterios para su elección" (p.74). En este sentido para el informe se consideró a los estudiantes del paralelo D del segundo año de bachillerato sección vespertina, que estuvo conformado por 40 estudiantes. 


\section{Tabla 1}

Población de la investigación

\begin{tabular}{|c|c|c|}
\hline Género & 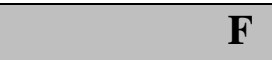 & $\%$ \\
\hline Varones & 23 & 57.5 \\
\hline Mujeres & 17 & 42.5 \\
\hline Total & 40 & 100 \\
\hline
\end{tabular}

Nota: Los datos fueron tomados de la secretaria de la Unidad Educativa.

\section{Criterio de inclusión y exclusión}

Criterios de inclusión: para la investigación se utilizó la totalidad de estudiantes de segundo año de bachillerato general unificado paralelo $\mathrm{D}$, quienes se conectan regularmente a las sesiones de aprendizaje, además de ser un grupo de estudiantes formado en donde las características son comunes. En este sentido Caballero (2014) indica que "estos criterios se precisan como aquellos elementos necesarios de la población seleccionada, por medio de este criterio el investigador logra responder la pregunta que se plantea en la investigación" (p.35).

Criterios de exclusión: Según los aportes de Salkind (1999) sostiene que son "conceptualizaciones predefinidas que sirven para distinguir a quienes no participaran en el estudio, estos criterios se forman por elegibilidad y se direccionan por el objetivo de estudio" (p.64). De acuerdo a ello se evita aquellos sujetos que no son viables para el seguimiento de la investigación, en base a lo expuesto la población que se excluye es toda la sección matutina y los demás cursos de la sección vespertina que no pertenecen a segundo curso de bachillerato.

\section{Muestra}

En palabras de Hernández, Mendoza (2018) la muestra se caracteriza "como un elemento pequeño o subconjunto que es seleccionado en la población", además de precisar que estos grupos constituidos son intactos, por consiguiente, la muestra toda vez que son grupos que no podemos desintegrar se considera a la totalidad de la población, es por ello que quedo establecida de la siguiente manera. 


\section{Tabla 2}

Muestra de la investigación

\begin{tabular}{lcc}
\hline Género & F & \% \\
\hline Varones & 23 & 57.5 \\
Mujeres & 17 & 42.5 \\
\hline Total & $\mathbf{4 0}$ & $\mathbf{1 0 0}$ \\
\hline
\end{tabular}

Nota: Los datos fueron tomados de la secretaria de la Unidad Educativa.

\section{Muestreo}

El muestreo en esta investigación es no probabilístico por conveniencia. Muestreo se utilizó debido a que el investigador es parte de la institución y por diversos motivos favorables se eligió la muestra. Además, se considera como el proceso que no pretende utilizar ningún recurso o metodología estadística para seleccionar la muestra, se ejecuta al considerar elementos imprescindibles y a conveniencia del investigador. (Latorre, Delio del Rincón, Arnal, 1996)

\section{Unidad de análisis}

Se enfoca en los estudiantes elegidos en la muestra, los mismos que son adolescentes entre 15 a 18 años.

\subsection{Técnicas e instrumentos de recolección de datos}

Como parte de las técnicas para el recojo de los datos se consideró a la encuesta, la misma que se conceptualiza en un documento para recolectar datos sobre un asunto determinado, con una población establecida con algunas características en específico. (Carrasco, 2009)

\section{Instrumentos de recolección de datos.}

Se hace imprescindible el uso de un instrumento para el recojo de los datos, por consiguiente, se optó por el cuestionario que conceptualizado por (Hernández et al., 2013). Considera como un acumulado de interrogantes de forma estructurada y orientadas a obtener información de vital importancia para el proceso de investigación. Es importante hacer referencia que el instrumento fue elaborado producto de un riguroso proceso de operacionalización de variables:

Por lo descrito anteriormente el cuestionario está estructurado en 10 preguntas que corresponde a la variable aprendizaje de matemática, las mismas que están en relación a los indicadores, dimensiones y la variable en estudio. Por otro lado, la estrategia didáctica no se elaboró instrumento toda vez que es la propuesta y se constituye en la solución a un problema que ha sido diagnosticado por el instrumento elaborado previamente. 


\section{Validez del instrumento}

De acuerdo a este acápite tenemos que se optó por la validez del contenido del instrumento, la misma que fue realizada por cinco jueces expertos, quienes evaluaron la relevancia y pertinencia de ítems e indicadores. la validez de contenido no se manifiesta de forma numérica aplicando coeficientes, niveles de significancia o índices, esta evaluación fue efectuada por medio de del juicio en forma subjetiva, es a este proceso que lo denomina validez por juicio de expertos, el proceso de esta valoración se realiza con la identificación de los profesionales en el ámbito de la investigación con el propósito de evaluar las preguntas del instrumento en relación de ser relevantes con la población, una redacción clara en tendencia de la formulación de preguntas, cada juez recibió la información necesaria y suficiente en virtud del objetivo del cuestionario, caracterización del contenido, operacionalidad, el instrumento debe estar categorizado de forma que sea congruente, tenga dominio del estudio, claro, parcial y observacional; como se evidencia este proceso no se lo realiza de forma cuantitativa por medio de análisis numérico, índices, coeficientes o significancia. (Lakatos, 1998)

De acuerdo a estos criterios de evaluación, los expertos realizaron un trabajo consiente y responsable, demostrando su profesionalismo al momento de deliberar el mismos que concluye como un valor aceptable tal como se manifiesta en los anexos.

\section{Confiabilidad del instrumento.}

En el mismo sentido la confiabilidad de la encuesta se procesó utilizando el programa SPSS y se obtuvo el alfa de Cronbrach, dando como resultado un 0,867 como se muestra en los anexos. Se comprende por confiabilidad a la precisión de la magnitud de alguna característica o aspecto, alfa de Cronbach generalmente es utilizado por su disponibilidad de escalas, al aplicar el sistema SPSS se puede calcular este coeficiente de forma sencilla y ágil, colabora con la decisión que se debe tomar al aplicar el cuestionario de investigación. (Valderrama, León, 2009).

\section{Procedimientos}

Teniendo en cuenta que las clases se desarrollan de forma virtual, entonces para la aplicación del instrumento se desarrolló en un formulario de google forms, a los estudiantes se envió el link del formulario por medio de un grupo en la red social WhatsApp; antes de aplicar la encuesta se gestionó la autorización de MSc. Gladys Mora 
Peña, rectora de la Unidad Educativa Fiscal Vicente Rocafuerte, el documento que autoriza la aplicación del instrumento se encuentra en anexos.

Una vez que se cuenta con la autorización y el consentimiento informado a los participantes se aplicó el formulario para obtener la información en relación al aprendizaje de las matemáticas, posteriormente se procesó los resultados, estableciendo tablas de frecuencia e interpretaciones de los resultados.

Para la identificar estrategias para fortalecer el aprendizaje de matemáticas se utilizó el análisis documental esta recopilación se realizó de los diferentes repositorios institucionales de universidades, para luego categorizar las estrategias que se utilizan en la enseñanza de las matemáticas, del mismo modo para identificar las teorías que las sustentan

\section{Método de análisis de datos}

En concordancia a lo solicitado los métodos que se han utilizado para el recojo de la información son parte de la estadística descriptiva debido a que se diagnosticó la variable en estudio; para ello se consideró tablas de frecuencia en donde se registran los calificativos de los estudiantes de la institución en mención; en este sentido (Ary et al., 1989) indican que este acápite se utilizó tablas de frecuencia y figuras en donde se mide las dimensiones de la variable dependiente.

En relación al análisis documental, estos documentos se procesaron identificando frases similares o estandarizando ciertas frases, lo que permitiría describir y obtener una tabla de todas las investigaciones realizada en referencias a las estrategias utilizadas en la enseñanza de las matemáticas.

\section{Aspectos éticos}

En relación a la parte ética se consideró que todo estudio de carácter científico es importante enfatizar en los criterios que garanticen el beneficio de quienes participan como muestra de estudio y sobre todo que no se presente ningún tipo de prejuicio para quienes participan ni para el contexto social. Es por ello que se considera el aporte de Sañudo (2006) manifestó que todo personal a cargo de la pesquisa muestra mucho respeto por cada colaborador, dado que se trata de individuos valiosos con autonomía para decidir su participación en el proyecto.

Por otro lado, es vital mantener en reserva los nombres de los informantes, y que los datos no sean utilizados para otras investigaciones o fines parecidos, es oportuno manifestar 
que la información utilizada en la investigación fue respetando la autoría de los investigadores consultados.

\section{3.-. RESULTADOS Y DISCUSIÓN}

Para el diagnóstico del uso de las TAC en las clases de matemáticas por los estudiantes de segundo bachillerato, Unidad Educativa Vicente Rocafuerte, de Ecuador durante el año 2020, en el ámbito académico y recursos tecnológicos, se aplicó una encuesta por 10 de preguntas a 40 estudiantes, teniendo como resultado lo siguiente.

\section{AMBITO ACADÉMICO}

\section{Tabla 3}

Recursos tecnológicos para enseñar matemáticas

\begin{tabular}{lcc}
\hline \multicolumn{1}{c}{ Repuestas } & Frecuencias & \% \\
\hline Siempre & 6 & 15.00 \\
Casi siempre & 10 & 25.00 \\
Algunas veces & 4 & 10.00 \\
Nunca & 20 & 50.00 \\
\hline Total & $\mathbf{4 0}$ & $\mathbf{1 0 0 . 0 0}$ \\
\hline
\end{tabular}

En la siguiente figura se observa que el $60 \%$ de los estudiantes declararon que los docentes no utilizan recursos tecnológicos para enseñar temas relacionados con la matemática.

\section{Figura 1}

Resultados de recursos tecnológicos para enseñar matemáticas

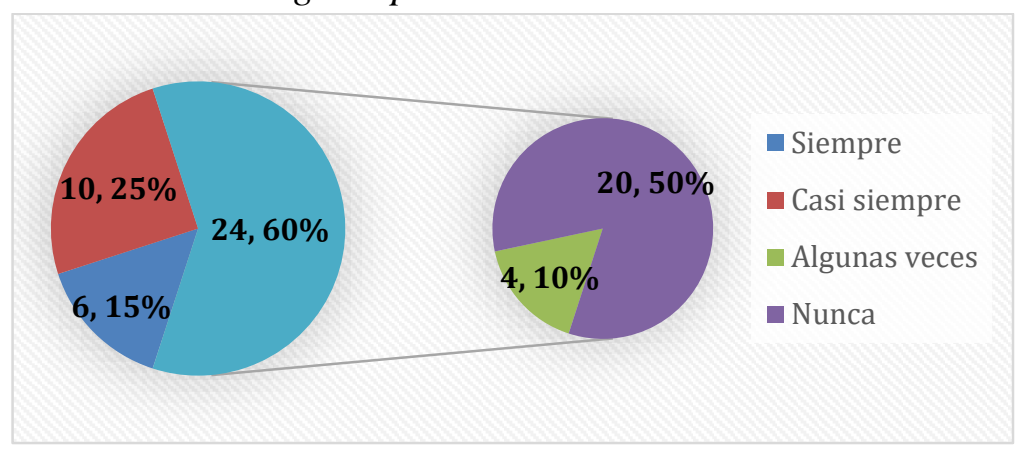

Tabla 4

Importancia de utilizar software educativo para su aprendizaje

\begin{tabular}{lcc}
\hline Repuestas & Frecuencias & \% \\
\hline Siempre & 24 & 60.00 \\
Casi siempre & 4 & 10.00 \\
Algunas veces & 11 & 27.50 \\
Nunca & 1 & 2.50 \\
\hline Total & $\mathbf{4 0}$ & $\mathbf{1 0 0 . 0 0}$ \\
\hline
\end{tabular}


En la siguiente figura se observa que el $70 \%$ de los estudiantes indicaron que es muy importante la utilización de software educativo para su aprendizaje.

\section{Figura 2}

Resultados de la Importancia de utilizar software educativo para su aprendizaje

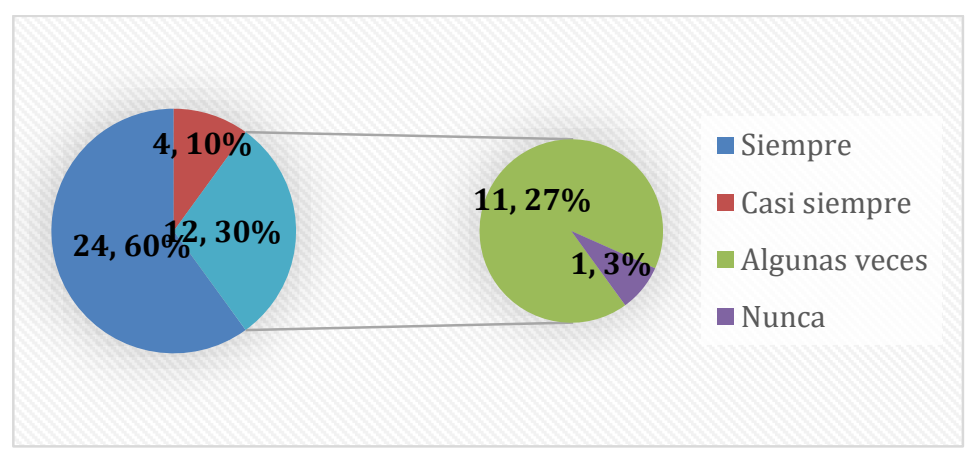

\section{Tabla 5}

Clases de matemáticas interesantes

\begin{tabular}{|c|c|c|}
\hline Repuestas & Frecuencias & $\%$ \\
\hline Siempre & 8 & 20.00 \\
\hline Casi siempre & 9 & 22.50 \\
\hline Algunas veces & 5 & 12.50 \\
\hline Nunca & 18 & 45.00 \\
\hline Total & 40 & 100.00 \\
\hline
\end{tabular}

En la figura se observa que el $58 \%$ de los estudiantes manifestaron que las clases de matemáticas son pocas interesantes, de los cuales el $45 \%$ indicaron que nunca y el $13 \%$ algunas veces son interesantes las clases de matemáticas

\section{Figura 3}

Resultados Clases de matemáticas interesantes

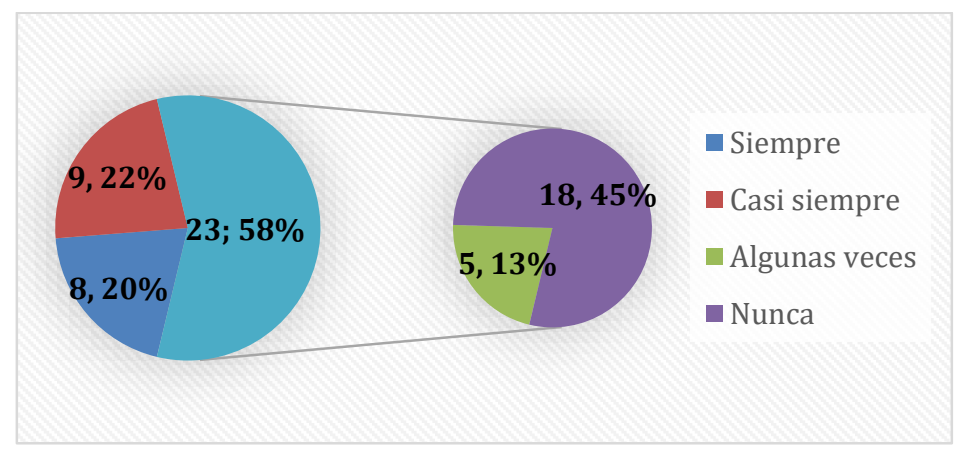




\section{Tabla 6}

Uso vía e-mail, para expresarle ideas

\begin{tabular}{lcc}
\hline \multicolumn{1}{c}{ Repuestas } & Frecuencias & \% \\
\hline Siempre & 1 & 2.50 \\
Casi siempre & 1 & 2.50 \\
Algunas veces & 8 & 20.00 \\
Nunca & 30 & 75.00 \\
\hline Total & $\mathbf{4 0}$ & $\mathbf{1 0 0 . 0 0}$ \\
\hline
\end{tabular}

Del total de encuestados, el 95\% manifestaron que no se dirigen vía e-mail para expresarle ideas a sus docentes, para aclarar dudas prefieren hacerlo directamente.

\section{Figura 4}

Resultados Uso vía e-mail, para expresarle ideas

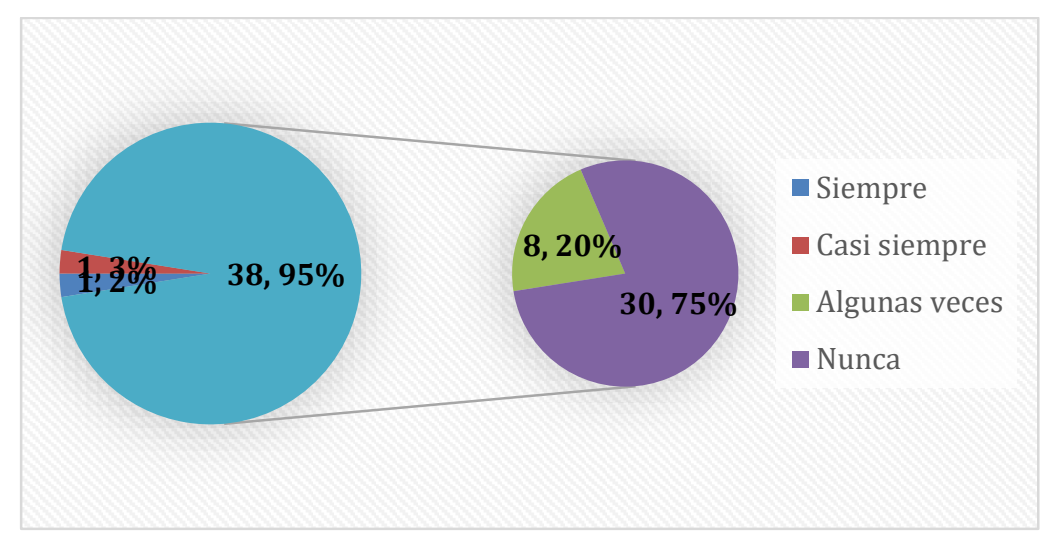

\section{Tabla 7}

Comunicación online con compañero

\begin{tabular}{lcc}
\hline Repuestas & Frecuencias & $\%$ \\
\hline Siempre & 4 & 10.00 \\
Casi siempre & 5 & 12.50 \\
Algunas veces & 18 & 45.00 \\
Nunca & 13 & 32.50 \\
\hline Total & $\mathbf{4 0}$ & $\mathbf{1 0 0 . 0 0}$ \\
\hline
\end{tabular}

El 78\% de los encuestados no establecen comunicación usando la tecnología para realizar actividades académicas y aclarar dudas. 


\section{Figura 5}

Resultados Comunicación online con compañero

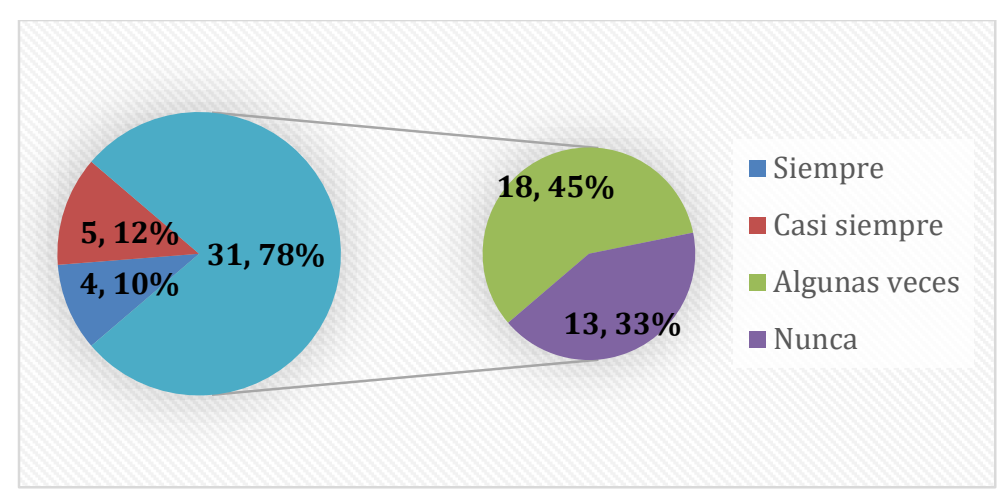

\section{RECURSOS TECNOLOGICOS}

\section{Tabla 8}

Utiliza computadora para realizar actividades académicas

\begin{tabular}{lcc}
\hline \multicolumn{1}{c}{ Repuestas } & Frecuencias & \% \\
\hline Siempre & 14 & 35.00 \\
Casi siempre & 10 & 25.00 \\
Algunas veces & 16 & 40.00 \\
Nunca & 0 & 0.00 \\
\hline Total & $\mathbf{4 0}$ & $\mathbf{1 0 0 . 0 0}$ \\
\hline
\end{tabular}

Como se observa en la siguiente figura, el $60 \%$ de los estudiantes manifestaron que utilizan computadora y/o otras tecnologías de información para realizar actividades de matemáticas

\section{Figura 6}

Resultados Utiliza computadora para realizar actividades académicas

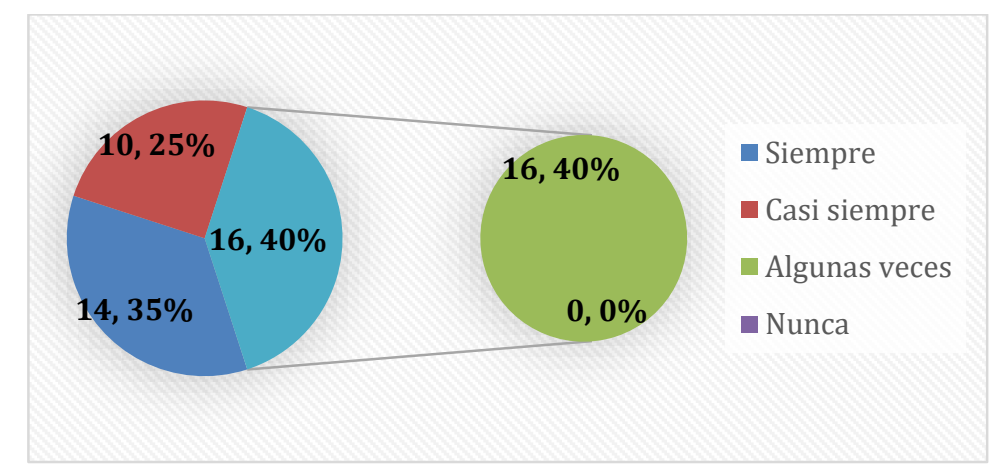




\section{Tabla 9}

Utiliza software Grafica Desmos para su proceso de aprendizaje

\begin{tabular}{|c|c|c|}
\hline Repuestas & Frecuencias & $\%$ \\
\hline Siempre & 1 & 2.50 \\
\hline Casi siempre & 4 & 10.00 \\
\hline Algunas veces & 14 & 35.00 \\
\hline Nunca & 21 & 52.50 \\
\hline Total & 40 & 100.00 \\
\hline
\end{tabular}

De los datos obtenidos en la figura siguiente se observa que el $88 \%$ de los estudiantes manifestaron que 14 utilizaron alguna vez y 21 manifestaron que nunca han utilizado el software educativo calculadora gráfica Desmos para su aprendizaje.

Figura 7

Resultados Utiliza software Grafica Desmos para su proceso de aprendizaje

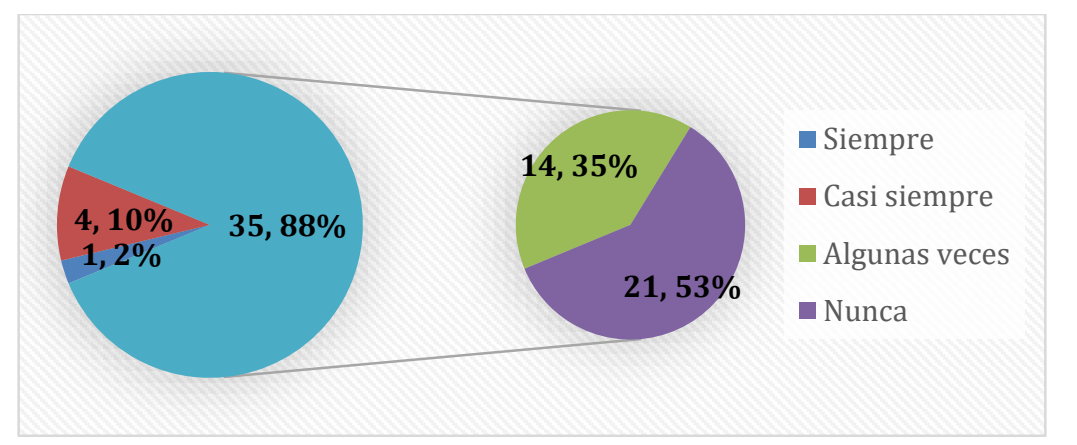

\section{Tabla 10}

Utiliza software GeoGebra para su proceso de aprendizaje

\begin{tabular}{|c|c|c|}
\hline Repuestas & Frecuencias & $\%$ \\
\hline Siempre & 1 & 2.50 \\
\hline Casi siempre & 7 & 17.50 \\
\hline Algunas veces & 11 & 27.50 \\
\hline Nunca & 21 & 52.50 \\
\hline Total & 40 & 100.00 \\
\hline
\end{tabular}

De los datos obtenidos, se observa en la siguiente figura que el $81 \%$ de los estudiantes manifestaron que 11 utilizaron alguna vez y 21 manifestaron que nunca han utilizado el software educativo GeoGebra para su aprendizaje. 


\section{Figura 8}

Resultados utiliza software GeoGebra para su proceso de aprendizaje

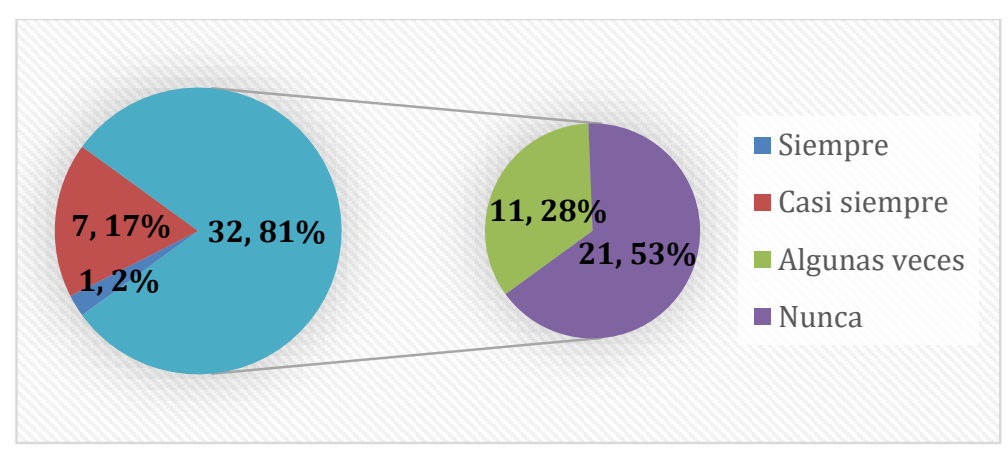

\section{Tabla 11}

Relaciona el contenido con la tecnología

\begin{tabular}{|c|c|c|}
\hline Repuestas & Frecuencias & $\%$ \\
\hline Siempre & 4 & 10.00 \\
\hline Casi siempre & 13 & 32.50 \\
\hline Algunas veces & 20 & 50.00 \\
\hline Nunca & 3 & 7.50 \\
\hline Total & 40 & 100.00 \\
\hline
\end{tabular}

Como se observa en la siguiente figura, el $58 \%$ de los estudiantes en alguna ocasión relacionan el contenido de la materia con la tecnología.

\section{Figura 9}

Resultados relaciona el contenido con la tecnología

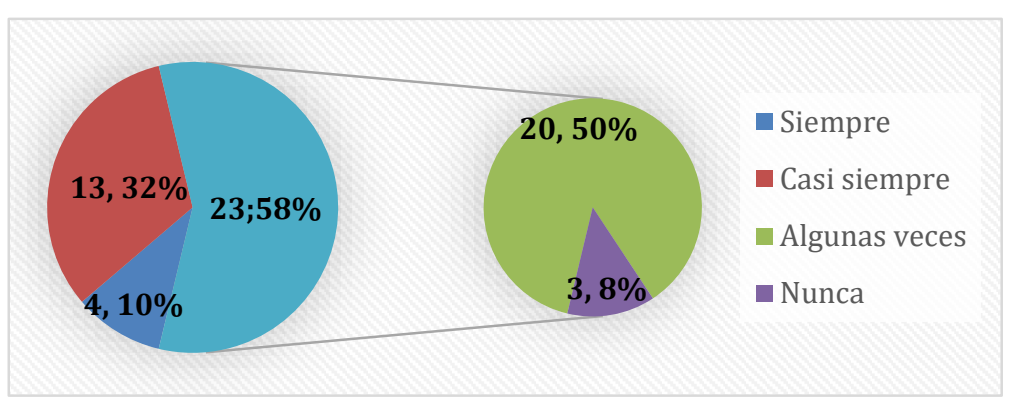

\section{Tabla 12}

Facilidad de resolver un problema utilizando la tecnología

\begin{tabular}{|c|c|c|}
\hline Repuestas & Frecuencias & $\%$ \\
\hline Siempre & 12 & 30.00 \\
\hline Casi siempre & 11 & 27.50 \\
\hline Algunas veces & 15 & 37.50 \\
\hline Nunca & 2 & 5.00 \\
\hline Total & 40 & 100.00 \\
\hline
\end{tabular}


El $57 \%$ de los estudiantes manifestaron utilizar la tecnología para resolver problemas matemáticos con gran facilidad.

\section{Figura 10}

Resultados facilidad de resolver un problema utilizando la tecnología

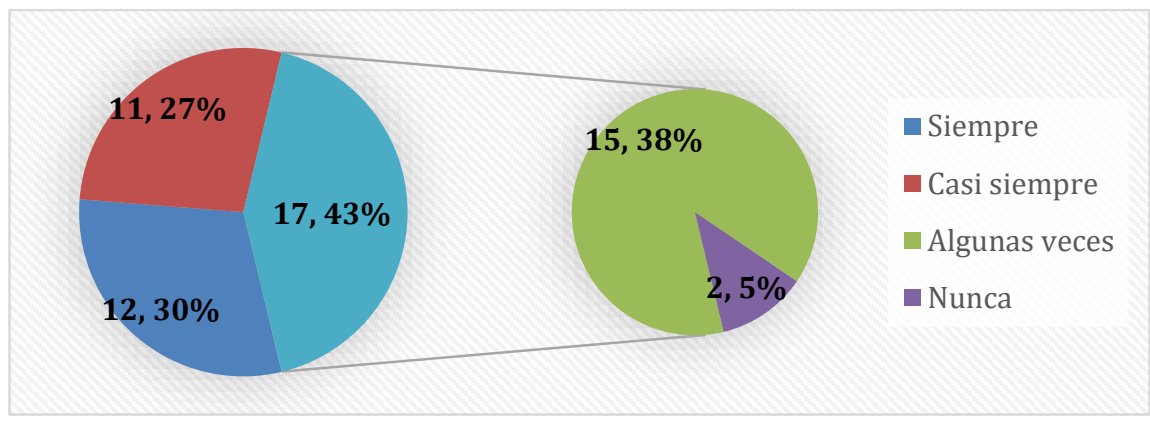

Para identificar las estrategias para fortalecer el aprendizaje de matemáticas se procedió al análisis documental de los antecedentes marco teórico y se sistematizó en la siguiente tabla.

Tabla 13.

Documentos revisados de diferentes trabajos de investigación

\begin{tabular}{|c|c|c|c|}
\hline Autores & $\begin{array}{l}\text { Objetivo de la } \\
\text { investigación }\end{array}$ & $\begin{array}{l}\text { Variables de } \\
\text { investigación }\end{array}$ & Resultados \\
\hline $\begin{array}{l}\text { Medina, } \\
\text { Pérez } \\
2021\end{array}$ & $\begin{array}{l}\text { Estimar las estrategias } \\
\text { heurísticas influyen en el } \\
\text { aprendizaje de } \\
\text { matemáticas } \\
\text { estudiantes de secundaria }\end{array}$ & $\begin{array}{l}\text { Estrategias } \\
\text { heurísticas } \\
\text { Aprendizaje } \\
\text { matemáticas }\end{array}$ & $\begin{array}{l}\text { La estrategia heurística permite } \\
\text { que se dé soluciones a los } \\
\text { problemas propuestos. }\end{array}$ \\
\hline $\begin{array}{l}\text { Reyes } \\
(2019)\end{array}$ & $\begin{array}{lrr}\begin{array}{l}\text { Determinar } \\
\text { aplicación si }\end{array} & \text { la } \\
\text { metodologías } & \text { de } \\
\text { puede mejorar las } & \\
\text { habilidades matemáticas }\end{array}$ & $\begin{array}{l}\text { ABP - } \\
\text { Habilidades } \\
\text { matemáticas }\end{array}$ & $\begin{array}{l}\text { La aplicación de las metodologías } \\
\text { de ABP si mejoran las habilidades } \\
\text { matemáticas de los estudiantes }\end{array}$ \\
\hline $\begin{array}{l}\text { Palomino } \\
\text { (2018) }\end{array}$ & $\begin{array}{l}\text { Determinar la relación } \\
\text { entre el aprendizaje } \\
\text { significativo y las } \\
\text { actitudes hacia las } \\
\text { matemáticas }\end{array}$ & $\begin{array}{l}\text { aprendizaje } \\
\text { significativo } \\
\text { y las actitudes } \\
\text { hacia las } \\
\text { matemáticas }\end{array}$ & $\begin{array}{l}\text { Los estudiantes tienen } \\
\text { dificultades para desarrollar las } \\
\text { operaciones matemáticas, además } \\
\text { de seguir procedimientos } \\
\text { sistemáticos en su desarrollo }\end{array}$ \\
\hline $\begin{array}{l}\text { Mora et } \\
\text { al. }(2021)\end{array}$ & $\begin{array}{l}\text { Proceso de las } \\
\text { competencias } \\
\text { matemáticas alcanzadas } \\
\text { por los estudiantes }\end{array}$ & & 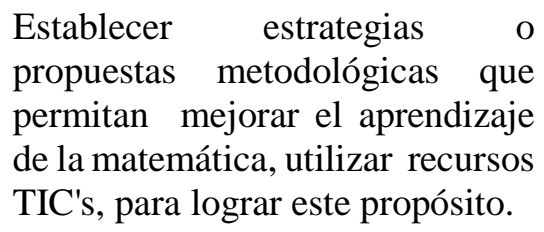 \\
\hline
\end{tabular}




\begin{tabular}{|c|c|c|c|}
\hline $\begin{array}{l}\text { Sánchez } \\
\text { (2020) }\end{array}$ & $\begin{array}{l}\text { Aplicar Gamificación } \\
\text { para fortalecer el } \\
\text { aprendizaje significativo } \\
\text { en matemáticas }\end{array}$ & $\begin{array}{l}\text { Gamificación } \\
\text { Aprendizaje } \\
\text { significativo } \\
\text { en } \\
\text { matemáticas }\end{array}$ & $\begin{array}{l}\text { Utiliza la Gamificación como } \\
\text { estrategia de aprendizaje en el } \\
\text { proceso educativo para fortalecer } \\
\text { los estudios de matemáticas. }\end{array}$ \\
\hline $\begin{array}{l}\text { Macías, } \\
2017\end{array}$ & $\begin{array}{l}\text { Mejorar el desempeño } \\
\text { académico de los } \\
\text { estudiantes de lero BGU, } \\
\text { en función del desarrollo } \\
\text { de la competencia } \\
\text { matemática plantear y } \\
\text { resolver problemas, e } \\
\text { incrementar la } \\
\text { Gamificación como } \\
\text { estrategia }\end{array}$ & $\begin{array}{l}\text { Desempeño } \\
\text { académico } \\
\text { Gamificación }\end{array}$ & $\begin{array}{l}\text { La aplicación de la estrategia de } \\
\text { Gamificación como apoyo a las } \\
\text { clases presenciales y con un pape } \\
\text { protagónico en las clases } \\
\text { virtuales, ravorece } \\
\text { significativamente el desarrollo de } \\
\text { la competencia matemática } \\
\text { plantear y resolver problemas, } \\
\text { siempre y cuándo se armonice una } \\
\text { adecuada instrucción pedagógica } \\
\text { con los elementos del juego }\end{array}$ \\
\hline $\begin{array}{l}\text { Iquise } \quad y \\
\text { Rivera, } \\
2020\end{array}$ & $\begin{array}{l}\text { Analizar la importancia } \\
\text { de la Gamificación como } \\
\text { estrategia en el proceso } \\
\text { de enseñanza y } \\
\text { aprendizaje }\end{array}$ & $\begin{array}{l}\text { Gamificación } \\
\text { Estrategia } \\
\text { proceso } \\
\text { enseñanza } \\
\text { aprendizaje }\end{array}$ & $\begin{array}{l}\text { La Gamificación es importante y } \\
\text { beneficioso en lo que se refiere en } \\
\text { la enseñanza porque consigue } \\
\text { motivar a los estudiantes, siendo } \\
\text { buena alternativa para mejorar su } \\
\text { aprendizaje. }\end{array}$ \\
\hline $\begin{array}{l}\text { Zapata, } \\
2021\end{array}$ & $\begin{array}{l}\text { Propuesta de un sistema } \\
\text { tecnológico para mejorar } \\
\text { la formación de las } \\
\text { competencias numéricas } \\
\text { en los alumnos }\end{array}$ & $\begin{array}{l}\text { competencias } \\
\text { numéricas }\end{array}$ & $\begin{array}{l}\text { Alumnos presentan niveles bajos } \\
\text { en matemáticas lo cual evidencia } \\
\text { la necesidad de incorporar el } \\
\text { programa tecnológico para } \\
\text { aprender matemáticas jugando }\end{array}$ \\
\hline
\end{tabular}

Luego de realizar una exhaustiva revisión de literatura de las diferentes investigaciones de repositorios digitales, se identificaron las estrategias utilizadas que permitirán fortalecer los aprendizajes en las matemáticas:

Tabla 14.

Estrategias seleccionadas para ser considerada en el diseño del modelo

\begin{tabular}{ll}
\hline Estrategias & Autores \\
\hline Heurística & $\begin{array}{l}\text { (Medina \& Pérez 2021). La estrategia heurística permite que se dé } \\
\text { soluciones a los problemas propuestos. }\end{array}$ \\
\hline & $\begin{array}{l}\text { (Macías, 2017), La aplicación de la estrategia de Gamificación } \\
\text { como apoyo a las clases presenciales y con un papel protagónico } \\
\text { en las clases virtuales, favorece significativamente el desarrollo de } \\
\text { la competencia matemática: plantear y resolver problemas, siempre } \\
\text { Gamificación } \\
\text { y cuándo se armonice una adecuada instrucción pedagógica con los } \\
\text { elementos del juego }\end{array}$ \\
\hline Solución de problemas \\
(Pólya, 1974, p.55), El método de George Pólya consiste en \\
determinar estrategias y método para la solución de problemas de \\
matemática, es el arte de resolver, problemas que ayuda a los \\
estudiantes a resolver sus problemas de matemática.
\end{tabular}


En relación a la propuesta de estrategias de aprendizaje de las matemáticas se ha considerado los principios de las teorías del Conectivismo, Constructivismo, aprendizaje significativo y el método de Polya como bases filosóficas que la sustentan. Entre el conjunto de estrategias que fueron consideradas tenemos la gamificación, la simulación y resolución de problemas utilizando las tecnologías como el software GeoGebra como un instrumento en la enseñanza y aprendizaje de la matemática

Figura 11 Modelo propuesto de estrategias didácticas

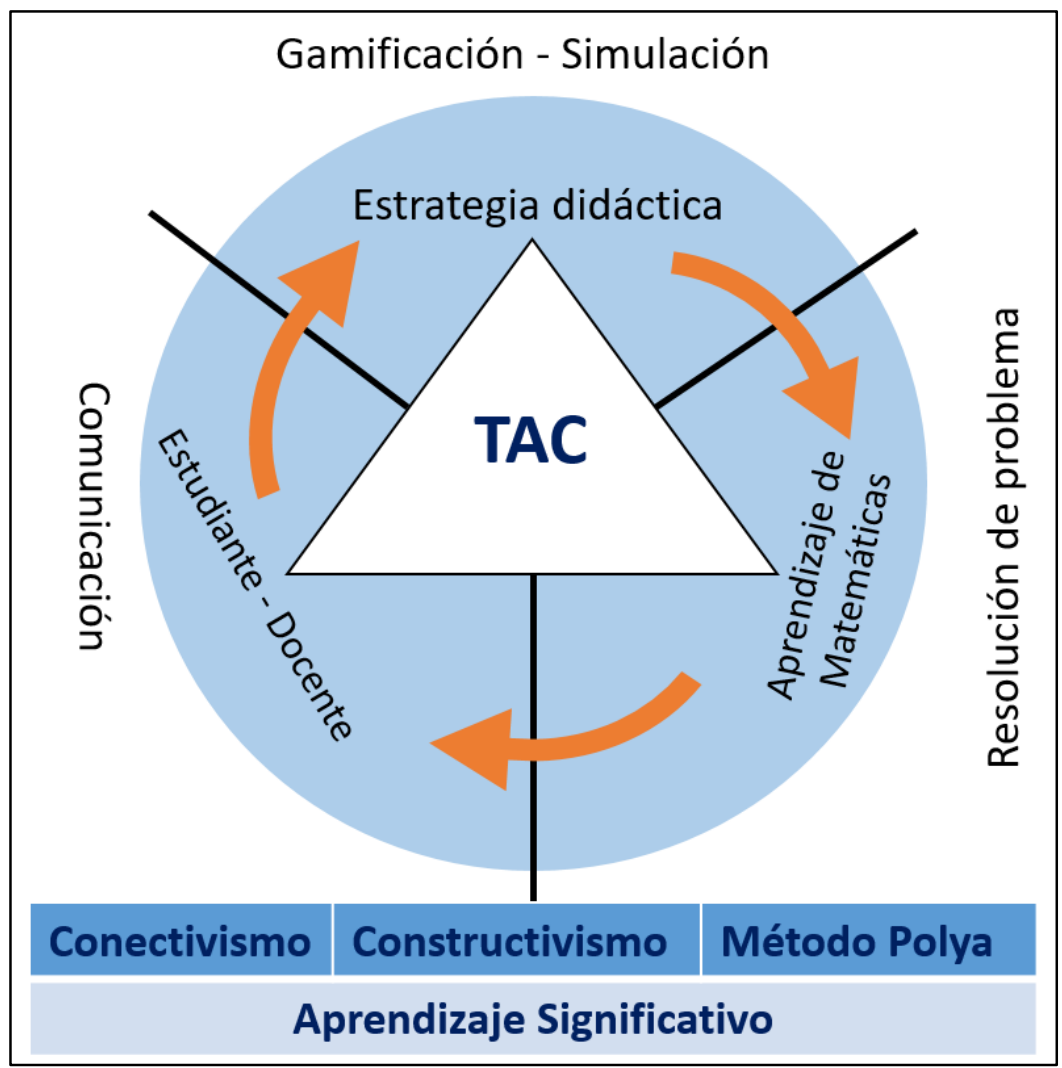

\section{DISCUSIÓN}

El objeto de estudio en esta investigación fue proponer un modelo de estrategia didáctica para fortalecer el aprendizaje de matemáticas en los estudiantes de 2 do año de bachillerato de la sección vespertina paralelo D, en la Unidad Educativa Fiscal Vicente Rocafuerte de Guayaquil. Luego del análisis realizado se procedió con la aplicación del instrumento de investigación, de forma virtual en el marco de la pandemia, con los resultados se analizó el estado en que se encuentra el proceso de aprendizaje en matemáticas, se encontró que los docentes no utilizan las tecnologías así como tampoco utilizan estrategias para fortalecer la enseñanza de las matemáticas, con estos resultados se determina que es 
necesaria la aplicación de un modelo de estrategia didáctica para mejorar el aprendizaje en matemáticas, lo cual se encuentra en la tablas 1,2 y 3. Lo argumentado se corrobora con lo expresado por Orellana (2016) en su publicación denominada "La estrategia didáctica y su uso dentro del proceso de enseñanza y aprendizaje en el contex to de las bibliotecas escolares" donde manifiesta que, una estrategia didáctica es la herramientas que facilita el desarrollo del aprendizaje con base en los contenidos de un programa o planificación, para llevarlos hacia la comprensión de los conceptos y su respectiva definición, con este proceso se traslada la información de forma eficaz aplicando los elementos que admiten la fluidez del aprendizaje, cada alumno posee conocimiento elemental en base a alguna información estos se deben relacionar con aquello que se conoce para fortalecer el tema de estudio e intercambiar ese aprendizaje en equipo con finalidad de construir nuevos aprendizajes.

Lo referido en la párrafo anterior, por el autor y en concordancia con el objetivo propuesto en la investigación, el modelo de estrategia didáctica es un valioso aporte con el cual se mejorará los medios utilizados por los docentes en vías de fortalecer el proceso de aprendizaje con una dinámica de enseñanza centrada en el educando, en conjunto con los medios que ya son aplicados por los educadores, esta propuesta se suma para concretar una comprensión con mayor solides y sobre todo en busca de aprendizaje sostenible, lo cual logrará que el estudiante active su memoria de trabajo, genere curiosidad y motivación por el aprendizaje numérico, debilitando el viejo paradigma insertado de forma generacional y sus fundamento sobre el temor hacia matemáticas y el esquema de que la disciplina es complicada y sin utilidad.

En el mismo contexto, la fiabilidad, consistencia y validez del instrumento de investigación fue sometido a la valoración mediante análisis por juicio de experto y a su vez por estadística descriptiva mediante el software, SPSS V25. La validez del contenido fue analizado por 5 profesionales con grado académico de doctor, quienes examinaron, comprobaron la pertinencia entre la variable de investigación sus dimensiones, estas con los indicadores y a su vez con los criterios que componen el cuestionario, el dictamen de los expertos fue de muy bueno, lo cual es afirmado por Ruiz (2013) en la publicación de su libro titulado "Instrumentos y Técnicas de Investigación Educativa Un Enfoque Cuantitativo y Cualitativo para la Recolección y Análisis de Datos" done expresa que, la validez de contenido no se manifiesta de forma numérica o por medio de coeficientes y 
niveles de significancia, por ser una estimación subjetiva se debe realizar por medio del juicio de aquí su denominación validez de contenido mediante juicio de expertos, para lo cual se debe seleccionar los profesionales en el ámbito de la investigación con el propósito de evaluar las preguntas del instrumento donde se confirma su relevancia con la muestra de estudio, debe tener redacción clara en tendencia de la formulación de las preguntas, cada juez debe recibir la información necesaria y suficiente en virtud del objetivo del cuestionario, caracterización del contenido, operacionalidad, el instrumento debe estar categorizado de forma que sea congruente con dominio en el estudio.

Es así como la fiabilidad del instrumento luego del análisis descriptivo en el SPSS V25, arrojó un coeficiente de 0,867 estimado como nivel bueno, el instrumento para el estudio se compuso por 2 dimensiones y 10 ítems en escala de Likert y aplicado de forma virtual. Esto es corroborado por Illescas-Cárdenas et al. (2020) quien de forma muy similar en su publicación "Aprendizaje Basado en Juegos como estrategia de enseñanza de la Matemática" presenta un cuestionario configurado con 11 items y desarrollado en google en escala de Likert, la fiabilidad del por alfa de Cronbrach obtuvo un coeficiente de 0,86. Lo expuesto es confirmado por Rodríguez-Rodríguez y Reguant-Álvarez (2020) quienes en su artículo que lleva como título "Calcular la fiabilidad de un cuestionario o escala mediante el SPSS: el coeficiente alfa de Cronbach“" manifiesta que, se comprende por confiabilidad a la precisión de la magnitud de alguna característica o aspecto, alfa de Cronbach generalmente es utilizado por su disponibilidad de escalas, al aplicar el sistema SPSS se puede calcular este coeficiente de forma sencilla y ágil, colabora con la decisión que se debe tomar al aplicar el cuestionario de investigación.

El modelo de estrategias didácticas propuesto se estructura en desarrollar temas tales como la Gamificación que según (Valda \& Arteaga, 2015) es el uso de mecánicas, componentes y dinámicas propias de los juegos, esto hace que las clases sean más dinámicas simplificando actividades complejas y crea una retroalimentación positiva haciendo que los estudiantes fomenten la comunicación, compañerismo y de esta manera cumplir con las metas y por el otro lado los docentes pueden realizar el seguimiento sobre las actividades realizadas y poder fortalecer su aprendizaje de una manera continua. También se ha considerado el método de Pólya que según Casimiro, 2017, es un método heurístico donde uno de sus objetivos principales es desarrollar nuevos procesos para la solución de problemas matemáticos en cuatro pasos como lo menciona (Carruthers, 
2016), la resolución de problemas se "compone de cuatro elementos como son explorar para entender el problema, hallar la estrategia apropiada, aplicar estrategia para resolver y revisar de principio a fin para comprobar la solución", de manera que contribuyan al razonamiento lógico y así facilitar el proceso de aprendizaje en los estudiantes.

Además es importante en este contexto incorporar la TICs, según (Monzón, 2020), “Ellas constituyen medios facilitadores para la enseñanza y aprendizaje de la matemática porque permite hacer simulaciones, visualizar gráficas en 2D y 3D, realizar cálculos con rapidez y exactitud". (p. 30). Por eso es importante incluir en este modelo el uso del GeoGebra como un instrumento en la enseñanza y aprendizaje de la matemática puede ayudar a generar imágenes visuales, organizar datos y realizar cálculos. Cuando disponen de herramientas tecnológicas, los estudiantes pueden focalizar su atención en procesos de toma de decisiones, reflexión, razonamiento y resolución de problemas” (Santo, 2001, p. 247).

\section{4 .- CONCLUSIÓN}

- Del diagnóstico en relación al uso de las TAC en las clases de matemáticas en estudiantes de segundo bachillerato de la Unidad Vicente Rocafuerte Ecuador se concluyó que en el ámbito académico. Que los docentes más del 60\% no utilizan recursos tecnológicos para para enseñar temas relacionado a las matemáticas, sin embargo más del $95 \%$ No se dirige a su profesor, vía e-mail, para expresarle ideas que no se atrevería a decirle cara a cara en clase, así como el $78 \%$ de los estudiantes no establecen comunicación entre compañeros para alguna actividad académica, en relación a los recursos tecnológicos, el $88 \%$ no ha tenido alguna experiencia con el uso de software educativo Calculadora Gráfica Desmos para su proceso de aprendizaje y $81 \%$ tampoco en el uso del GeoGebra.

- Se Identificaron las estrategias para fortalecer el aprendizaje de matemáticas como estrategias heurísticas, que permitirán se soluciones problemas en el aprendizaje de las matemáticas, la gamificación como apoyo a las clases presenciales y virtuales para el fortalecimiento de la competencia de matemática, siempre y cuando se armonice una adecuada instrucción pedagógica.

- Se diseñó un modelo de estrategia didáctica que fortalecerá el aprendizaje de matemática en estudiantes de segundo bachillerato de la Unidad Educativa Vicente Rocafuerte de Ecuador. Se inició con el diagnostico que fue vital para identificar lo 
académico y tecnológico, luego se identificaron las estrategias utilizadas en la enseñanza de las matemáticas, logrando considerarlas en el en el diseño del modelo, así mismo se fortaleció con las teorías del Conectivismo a fin de establecer la comunicación basada en nodo entre estudiantes, docentes y así tener una comunicación constante, del mismo modo se consideró la teoría del constructivismo y el método de Polya, utilizando las tecnologías como el software GeoGebra como un instrumento en la enseñanza y aprendizaje de la matemática, buscando en el modelo de estrategias didácticas fortalecer el aprendizaje en las matemáticas a los estudiantes de la unidad educativa Vicente roca Fuerte de Ecuador.

\section{RECOMENDACIONES}

El desarrollo de la enseñanza-aprendizaje de contenidos matemáticos mediante la aplicación de actividades estratégicas, deben lograr objetivos tales como despertar el interés en la asimilación de conocimientos en el estudiante, una actitud positiva para la formación y capacitación que genere competitividad en el futuro bachiller. Se sugiere una constaste comunicación entre sus docentes, estudiantes para lograr una comunicación exitosa en cada instancia de su labor académica, así como usar software educativo como la Calculadora Gráfica Desmos y GeoGebra para el proceso de aprendizaje.

Se sugiere optimizar las estrategias didácticas en el segundo año de bachillerato del plantel con el fin de mejorar el aprendizaje matemático del estudiante de bachillerato. Incorporando estrategias como heurísticas, gamificación, resolución de problemas y utilizar recursos tecnológicos como softwares educativos con el propósito de comprobar la solución de las actividades propuesta y así fortalecer su aprendizaje de las matemáticas.

Un modelo de estrategias didácticas, debe ser diseñado, según el nivel académico a ser aplicado, con el fin de ser utilizado tanto por profesores como por estudiantes. Así, comparando los resultados por dimensiones, se sugiere que el equipo directivo del centro educativo debe mejorar sus estrategias en cuanto a la dimensión de recursos tecnológicos usando software educativo.

Es imperante en la actualidad el uso de las herramientas tecnológicas, donde la avalancha de información, se encuentra al alcance en todos, aspecto, lo que debería ser aprovechado por los docentes, adaptando esta información de manera estratégica y didáctica para 
facilitar el aprendizaje matemático, donde el docente tendría el rol fundamental de orientador de la información para lograr su asimilación.

\section{LISTA DE REFERENCIAS}

Alarcón, R., Flores, H. (2021). Aplicación de algoritmos etnomatemáticos en el aprendizaje significativo de estudiantes universitarios. Innova Research Journal. 6(1), 195-215. https://doi.org/10.33890/innova.v6.n1.2021.1522

Andrew, P. y Xenophontic, C. (2015). Analyzing the relationship between the problemsolving-related beliefs, competence and teaching of the three Cypriot primary teachers. Journal of Mathematics Teacher Education, 18(4), 299-325. http://www.diva-portal.org/smash/record.jsf?pid=diva2\%3A905602\&dswid=7790

ARy, D., Jacobs, L., Razavieh, A. (1989). Introducción a la investigación pedagógica. Segunda edición. México. McGRAW-HILL

Ausubel, D. (1978). Adquisición y retención del conocimiento: Una perspectiva cognitiva. Tercera edición. Barcelona. España: Paidós.

Barrera, F. y Reyes, A. (2017). Tareas con diversas soluciones: estructuras conceptuales en profesores de matemáticas. Revista Electrónica de Investigación Educativa, 19(1), 110-122. DOI: https://doi.org/10.24320/redie.2017.19.1.971

Borges, S., Durelli, V., Reis, H., Bittencourt, I., Mizoguchi, R., \& Isotani, S. (octubre de 2017). Selecting Effective Influence Principles for Tailoring Gamification-Based Strategies to Player Roles. doi: 10.5753/cbie.sbie.2017.857

Buendía, L. (2001). La ética en la investigación Educativa. http://normaldemompox.tripod.com/documentos/laetica_en_la_investigacion_educativa.pdf

Caballero, A. (2014). Metodología integral innovadora para planes y tesis. La Metodología del cómo formularlos. México.

Carrasco, S. (2009). Metodología de la investigación científica. Lima, Perú: San Marcos.

Carruthers, P. (2016). La evolución de la memoria de trabajo. http://www.centrolombardo.edu.mx/ludus-vitalis/la-maquinaria-mental-humananum-40-2013/la-evolucion-de-la-memoria-de-trabajo-ludus-vitalis/

Casimiro, M. (2017). Método de Pólya en la resolución de problemas de ecuaciones (Tesis de grado, Universidad Rafael Landívar). 
http://recursosbiblio.url.edu.gt/tesiseortiz/2018/05/86/Casimiro-Maria.pdf

Chiroque, S., Valer, L. (1998). Didáctica General. Lima (Perú): Facultad de Educación UNMSM.

CONCYTEC (2018). Reglamento de calificación, clasificación y registro de los investigadores del sistema nacional de ciencia, tecnología e innovación tecnológica - reglamento Renacyt. https://portal.concytec.gob.pe/images/renacyt/reglamento_renacyt_version_final. pdf

Cruz, D. (2014). Despelling the notion of inconsistencies in teachers' mathematics beliefs and practices: A 3-year case study. Journal of Mathematics Teacher Education, 18, 173-201. https://link.springer.com/article/10.1007/s10857-0149276-5

Cullen, C. (2007). The Suàn shù sh, 'Writings on reckoning': Rewriting the history of early Chinese mathematics in the light of an excavated manuscript, Historia Mathematica, 34(1), 10-44. https://core.ac.uk/download/pdf/82177776.pdf

Culqui, R. (2019). Programa de estrategias lúdicas y su influencia en el desarrollo de competencias matemáticas en los estudiantes de $4^{\circ}$ grado del Nivel Primaria, Institución Educativa N. ${ }^{o}$ 15509, Talara - Piura, 2017. (Tesis doctoral, Universidad César Vallejo). https://repositorio.ucv.edu.pe/bitstream/handle/20.500.12692/28848/Culqui_GR E.pdf? sequence $=1 \&$ isAllowed $=\mathrm{y}$

Cunaprioaru, D. (2015). Problem Solving - Purpose and Means of Learning Mathematics in School, Procedia. Social and Behavioral Sciences. 191, 1859-1864. ISSN 1877-0428. https://doi.org/10.1016/j.sbspro.2015.04.332.

Díaz, V.R. y Parra, O (2014). Didáctica de las matemáticas y tecnologías de la información y la comunicación". En Colombia Revista Educación Y Desarrollo Social ISSN: 2011-5318 ed: Universidad Militar Nueva Granada. 8(1), 60- 81, 2014, DOI:10.18359/reds.295

El comercio (2019). Resultados de la evaluación PISA-D plantean varios retos en educación. https://www.elcomercio.com/actualidad/resultados-evaluacion-pisaretos-educacion.html 
Flores, L. (2011). Desarrollo de estrategias metodológicas constructivistas en el área de matemática en el sexto y séptimo año de EE.BB, mediante talleres o charlas pedagógicas a los niños de la Unidad Educativa “Francisco Calderón” Barrio Santán, Cantón Latacunga, en el año lectivo 2010- 2011. (Tesis de grado, Universidad Técnica de Cotopaxi). http://repositorio.utc.edu.ec/bitstream/27000/1441/1/T-UTC-1326.pdf

Gaitan, V. (2013). Gamificación en el aprendizaje divertido. Educativa. https://www.educativa.com/blog-articulos/gamificacion-el-aprendizajedivertido/

Gamarra, G., Berrospi, J., Pujay, O., Cuevas, R. (2008). Estadística e investigación. Lima. Perú: Editorial San Marcos.

Gardner H. (2016). Estructuras de la mente: La teoría de las inteligencias múltiples. Primera edición electrónica. México: Fondo de Cultura Económica.

Gerdes, P. (2007). Otthava, Fazer Cestos e Geometría na Cultura Makhuwa do Nordeste de Moçambique.Mozambique: Universidad de Lúrio Nampula. Obtenido de http://stores.lulu.com/pgerdes

Godino, J. D. Batanero, C. y Font, V. (2007). The onto-semiotic approach to research in mathematics education. ZDM. The International Journal on Mathematics Education, 39(1-2), 127-135. https://www.ugr.es/ jgodino/indice_eos.htm

Gómez, A. (2010). El proceso de enseñanza-aprendizaje de los conceptos de ordenación y combinación con estudiantes de educación superior: Un enfoque basado en la resolución de problemas (Tesis doctoral, Universidad Autónoma de Barcelona). https://www.educacion.gob.es/teseo/imprimirFicheroTesis.doidFichero=LYcyte LZX58\%3D

Hernández, E. (2008). El modelo constructivista con las nuevas tecnologías: aplicado en el proceso de aprendizaje. http://www.uoc.edu/rusc/5/2/dt/esp/hernandez.pdf

Hernández, R. Zapata, N., Mendoza, C. (2013). Metodología de la investigación para bachillerato. Enfoque por competencias. México: McGraw-Hill/Interamericana Editores.

Hernández, R., Mendoza, C. (2018). Metodología de la investigación. Las rutas cuantitativa, cualitativa y mixta. México: McGraw-Hill/Interamericana Editores. 
Hoon T. S., Kee, K. L., Singh P., (2013). Learning mathematics using a heuristic $\begin{array}{llll}\text { approach. } & \text { Revista } & \text { ScienceDirect. } & 90,\end{array}$ https://doi.org/10.1016/j.sbspro.2013.07.162

Hurtado de Barrera, J. (2008). Metodología de la investigación, una comprensión holística. Investigación proyectiva. http://investigacionholistica.blogspot.com/2008/02/la-investigacinproyectiva.html

Illescas-Cárdenas, R., García-Herrera, D., Erazo-Álvarez, C., \& Erazo-Álvarez, J. (2020). Aprendizaje Basado en Juegos como estrategia de enseñanza de la Matemática. CIENCIAMATRIA, 6(1), 533-552. https://doi.org/10.35381/cm.v6i1.345

Iquise, M. \& Rivera, L. (2020). La importancia de la gamificación en el proceso de enseñanza y aprendizaje. (Tesis de grado. Universidad San Ignacio de Loyola). http://repositorio.usil.edu.pe/bitstream/USIL/9841/1/2020_Iquise\%20Aroni.pdf

Laguzzi, G., Simón, J. (2018). Modos de organizar las clases: las secuencias didácticas. https://educrea.cl/modos-de-organizar-las-clases-las-secuencias-didacticas/

Lakatos, I. (1998). La metodología de los programas de investigación científica. Madrid: Alianza Universidad.

Latorre, A., Delio del Rincón, I., Arnal, J. (1996). Bases metodológicas de la investigación educativa. Barcelona: GR92.

León, D. (2018). Ansiedad escolar, Autoestima y aprendizaje matemático en estudiantes de primaria RED $N^{\circ} 01$ UGEL 02-Rímac 2016. (Tesis doctoral, Universidad César Vallejo).

https://repositorio.ucv.edu.pe/bitstream/handle/20.500.12692/22445/Le\%C3\%B $3 n \_J D . p d f ?$ sequence $=1$

Llatas, M. (2016). Programa de estrategias metodológicas para mejorar las habilidades matemáticas en los estudiantes del isep "Octavio matta contreras" de Cutervo, 2016. (Tesis doctoral, Universidad César Vallejo) https://repositorio.ucv.edu.pe/bitstream/handle/20.500.12692/2392/llatas_cm.pdf ?sequence $=1$

Macías, A. (2017). La Gamificación como estrategia para el desarrollo de la competencia matemática: plantear y resolver problemas. (Tesis de grado, 
Universidad

Casa

grande).

http://dspace.casagrande.edu.ec:8080/bitstream/ucasagrande/1171/2/Tesis1362M ACg.pdf

Martín, D.; Chacón, T., Curbera, G., Marcellán, F., Siles, M. (2020). Libro Blanco de las Matemáticas.

https://www.fundacionareces.es/recursos/doc/portal/2020/10/14/libro-blanco-delas-matematicas.pdf

Medina, V. H., y Pérez, M. A. (2021). Influencia de las estrategias heurísticas en el aprendizaje de la matemática. Innova Research Journal, 6(2), 36-61. https://doi.org/10.33890/innova.v6.n2.2021.1672

Medina, A., Pérez, L. y Campos, B. (2014). Elaboración de planes y programas de formación del profesorado en didácticas especiales. Madrid: UNED.https://dialnet.unirioja.es/servlet/libro? Código=567365

Minedu (2015).

Rutas

de

aprendizaje.

http://www.minedu.gob.pe/DeInteres/pdf/documentos-secundaria-matematicavii.pdf

Monzón, E. (2020). La influencia del uso del software GeoGebra en el logro del aprendizaje por competencias de matemática I en los estudiantes de la universidad nacional "Santiago Antúnez de Mayolo" - 2019. (Tesis de maestría, $\begin{array}{lllll}\text { Universidad de } & \text { San }\end{array}$ https://repositorio.usmp.edu.pe/handle/20.500.12727/6818

Mora, M., Calle, E., Guachún, P., Bernal, J. (2021). Investigación en educación matemática, en ecuador y la región caso Universidad de Cuenca. https://www.researchgate.net/publication/349251930_INVESTIGACION_EN_E DUCACION_MATEMATICA_EN_ECUADOR_Y_LA_REGION_CASO_UN IVERSIDAD_DE_CUENCA

Nureña, M. (2016). Estrategias didácticas para la enseñanza - aprendizaje. 3a. ed. Buenos Aires (Argentina): Kapelusz.

Orellana, C. (2017). La estrategia didáctica y su uso dentro del proceso de enseñanza y aprendizaje en el contexto de las bibliotecas escolares. e-Ciencias de la Información, 7(1), 134-154. http://dx.doi.org/10.15517/eci.v7i1.27241. 
Ortiz, C. (2016). Tutoría entre pares como una estrategia pedagógica universitaria. Educación $\quad y \quad$ Educadores, $\quad$ 309-325. https://www.redalyc.org/pdf/834/83421404006.pdf

Pacheco, A. (2014). Aprendiendo a enseñar, enseñando a aprender en la Universidad. Lima (Perú): Realidad Visual.

Palomino, J. (2018). Aprendizaje significativo y las actitudes hacia las matemáticas en estudiantes del VII ciclo, en la Institución Educativa 1227-Ate 2018. (Tesis doctoral, Universidad César https://repositorio.ucv.edu.pe/bitstream/handle/20.500.12692/17625/Palomino_ MJ.pdf?sequence=1\&isAllowed $=\mathrm{y}$

Parra, S. (2020). Resultados Educativos en el Ecuador: Examen Crítico a la Luz de los Exámenes Internacionales PISA. https://informativo.usfq.com/images/files/Koyuntura\%20Feb\%202020.pdf

Peralta, J. (2000). Principios didácticos e históricos para la enseñanza de la matemática. España: Huerga Fierro.

Piaget, J., Inhelder, B. (2007). La psicología del niño. 17ma edición. Madrid: Ed. Morata. Pisa (2020). Programa de evaluación de estudiantes internacionales. OCDE: Perú. Pólya, G. (1974). Cómo plantear y resolver problemas. México: Trillas.

Pouyamanesh, J., Firoozeh, L. (2013). Compared the Learning Outcomes of Students with Math in High and Low Frustration Tolerance. Procedia - Social and Behavioral Sciences, 84, 837-840. https://doi.org/10.1016/j.sbspro.2013.06.657

Revelo, J. (2020). Impacto del uso de las TIC como herramientas para el aprendizaje de la matemática de los estudiantes de educación media. Cátedra, 1(1), 70-91. https://doi.org/10.29166/catedra.v1i1.764

Reyes, J. (2019). El Aprendizaje basado en problemas para mejorar las capacidades de matemática en los estudiantes de formación tecnológica, Chocope 2019. (Tesis doctoral, Universidad César Vallejo). https://repositorio.ucv.edu.pe/bitstream/handle/20.500.12692/45018/Reyes_RJE _SD.pdf? sequence $=8 \&$ is Allowed $=\mathrm{y}$

Rodríguez-Rodríguez, J., y Reguant-Álvarez, M. (2020). Calcular la fiabilidad de un cuestionario o escala mediante el SPSS: el coeficiente alfa de Cronbach. REIRE 
Revista d'Innovación $i$ Recerca en Educación, 13(2), 1-13. https://doi.org/10.1344/reire2020.13.230048

Romo, A., Aguayo, L., Sánchez, M. (2020). Educación Matemática. Revista educación en matemáticas, $\quad 32(2)$. http://www.revista-educacionmatematica.org.mx/descargas/vol32/2/REM32-2.pdf

Ruiz, C. (2013). Instrumentos y Técnicas de Investigación Educativa Un Enfoque Cuantitativo y Cualitativo para la Recolección y Análisis de Datos. https://www.academia.edu/37886948/Instrumentos_y_Tecnicas_de_Investigaci \%C3\%B3n_Educativa_Carlos_Ruiz_Bolivar_pdf

Sáenz, E., Patiño, M., \& Robles, J. (2018). Development of mathematical competences in geometric thinking, through Polya's heuristic method. Panorama, 11(21), 5267. https://doi.org/10.15765/pnrm.v11i21.1055

Salkind, N. (1999). Métodos de investigación. México: Prentice Hall.

Sánchez, C. (2020). Gamificación personalizada para fortalecer aprendizajes significativos de la asignatura Matemática, en estudiantes de bachillerato de la ciudad de Guayaquil. (Tesis doctoral, Universidad César Vallejo). https://repositorio.ucv.edu.pe/handle/20.500.12692/54862

Santiváñez (2009). Documento de trabajo del curso de didáctica universitaria. Universidad Los Ángeles de Chimbote.

Santos, L. (2001). Potencial didáctico del software dinámico en el aprendizaje de las matemáticas. https://biblat.unam.mx/es/revista/avance-yperspectiva/articulo/potencial-didactico-del-software-dinamico-en-elaprendizaje-de-las-matematicas

Sañudo, 1. (2006). La ética en la investigación educativa: https://www.redalyc.org/pdf/4138/413835165006.pdf

Siemens, G. (2004). Connectivism: A Learning Theory for the Digital Age. http://www.elearnspace.org/Articles/connectivism.htm

Schoenfeld, A. (1985). Mathematical Problem Solving. Orlando: Academic Press.

INC. Academic Press. https://www.elsevier.com/books/mathematical-problemsolving/schoenfeld/978-0-12-628870-4

Tambunan, H. (2018). Impact of Heuristic Strategy on Students' Mathematics Ability in High Order Thinking. International Electronic Journal of Mathematics 
Education, 13(3), 321-328. https://doi.org/10.12973/iejme/3928

Tejeda, R. (2017). Estrategias heurísticas y clima escolar en el aprendizaje de la matemática en estudiantes de primer año de secundaria de la Red 2 de la UGEL 03- 2015. (Tesis doctoral, Universidad César Vallejo). https://hdl.handle.net/20.500.12692/8450

UNESCO (2020). ¿Qué se espera que aprendan los estudiantes de América Latina y el Caribe? Análisis curricular del Estudio Regional Comparativo y Explicativo (ERCE 2019). https://unesdoc.unesco.org/ark:/48223/pf0000373982

UNESCO (2021a). Matemáticas para un mundo mejor. https://es.unesco.org/news/14marzo-matematicas-mundo-mejor

UNESCO (2021b). Diagnóstico de aprendizajes de estudiantes bolivianos y llama a abordar las desigualdades en el sistema educativo. https://es.unesco.org/news/unesco-publica-diagnostico-aprendizajes-estudiantesbolivianos-y-llama-abordar-desigualdades

Valda, F. \& Arteaga C. (2015). Diseño e implementación de una estrategia de Gamificación en una plataforma virtual de educación. Fides et ratio, 9:81-90. http://www.scielo.org.bo/pdf/rfer/v9n9/v9n9_a06.pdf

Valderrama, S. (2013). Pasos para elaborar proyectos de investigación científica. Cuantitativa, cualitativa y mixta. Segunda edición. Lima, Perú: Editorial San Marcos.

Valderrama, S., León, L. (2009). Técnicas e instrumentos para la obtención de datos en la investigación científica. Lima: Editorial San Marcos.

Velásquez, V. (2018). Las estrategias didácticas y satisfacción académica de los estudiantes del primer ciclo de estudios de la Facultad de Pedagogía y Cultura Física de la Universidad Nacional de Educación Enrique. https://repositorio.une.edu.pe/handle/UNE/2430

Zapata, V. (2021). Propuesta JUMAT para mejorar el desarrollo de competencias matemáticas en estudiantes de una Institución Educativa Primaria del distrito de Morropón, 2020. (Tesis doctoral, Universidad César Vallejo). https://repositorio.ucv.edu.pe/bitstream/handle/20.500.12692/57482/Zapata_VVSD.pdf? sequence $=1 \&$ isAllowed $=\mathrm{y}$ 